\title{
Blood RNA expression profiles undergo major changes during the seventh decade
}

\author{
Marius Gheorghe ${ }^{1}$, Claudia Schurmann ${ }^{3,6}$ Marjolein J. Peters ${ }^{2}$, André G. \\ Uitterlinden ${ }^{2,5}$, Albert Hofmann ${ }^{2,5}$, Reiner Biffar ${ }^{7}$, Georg Homuth ${ }^{3}$, Uwe Völker ${ }^{3}$, \\ Joyce BJ van Meurs' ${ }^{2}$, Vered Raz \\ ${ }^{1}$ Department of Medical Informatics, Erasmus University Medical Center, Rotterdam, 3000 CA, The Netherlands \\ ${ }^{2}$ Department of Internal Medicine, Erasmus University Medical Center, Rotterdam, 3000 CA, The Netherlands \\ ${ }^{3}$ Department of Functional Genomics, Interfaculty Institute for Genetics and Functional Genomics, University Medicine \\ Greifswald, Greifswald, 17475 DE, Germany \\ ${ }^{4}$ Department of Human and Clinical Genetics, Leiden University Medical Centre, Leiden, 2300 RC, The Netherlands \\ ${ }^{5}$ Department of Epidemiology, Erasmus University Medical Center, Rotterdam, 3000 CA, The Netherlands \\ ${ }^{6}$ The Charles Bronfman Institute for Personalized Medicine, Genetics of Obesity and Related Metabolic Traits Program, Icahn \\ School of Medicine at Mount Sinai, New York, NY 10029, United States of America \\ ${ }^{7}$ Department of Prosthodontics, Gerodontology and Biomaterials, University Medicine Greifswald, Greifswald, 17475 DE, \\ Germany
}

Correspondence to: Vered Raz, email: v.raz@lumc.nl

Keywords: blood aging, population based studies, RNA expression profiles, sample weighting, b-spline regression model

Received: June 06, 2016

Accepted: September 05, 2016

Published: September 17, 2016

\section{ABSTRACT}

Genome-wide alterations in RNA expression profiles are age-associated. Yet the rate and temporal pattern of those alterations are poorly understood. We investigated temporal changes in RNA expression profiles in blood from population-based studies using a quadratic regression model. Comparative analysis between two independent studies was carried out after sample-weighting that downsized differences in sample distribution over age between the datasets. We show that age-associated expression profiles are clustered into two major inclinations and transcriptional alternations occur predominantly from the seventh decade onwards. The age-associated genes in blood are enriched in functional groups of the translational machinery and the immune system. The results are highly consistent between the two population-based studies indicating that our analysis overcomes potential confounders in population-based studies. We suggest that the critical age when major transcriptional alterations occur could help understanding aging and disease risk during adulthood.

\section{INTRODUCTION}

Age-dependent tissue deterioration characterizes physiological aging in multi-cellular organisms. Impaired maintenance of cellular homeostasis contributes to aging, partly caused by genome-wide transcriptional alterations affecting multiple gene networks [1]. In humans, the aging-associated gene network pattern is highly complex. Compared with model organisms, in humans the adulthood phase is much longer. Thus, slow progression in tissue and molecular alterations can contribute to the complexity of the aging process.

Most often, age-associated physiological and molecular alterations are extracted using linear regression models (few examples in: [2-7]). Linear regression assumes a constant change over time and therefore might be appropriate for organisms that aged over a short period [2]. In humans, however, adulthood spans from 50 to 80 years. It is very unlikely that the rate of age-associated changes progresses at a constant rate. The fitness of different regression models to describe age-associated physiological features demonstrated that a quadratic or a parabolic regression model are most suitable to describe age-associated changes [8]. We also reported that the fitness of a quadratic regression model to describe ageassociated changes in expression profiles is higher than linear or cubic regression models [9]. Quadratic models have fewer assumptions compared with a linear model. 
Moreover, a quadratic model could be employed to identify the age when major changes occur (named here age-position). Using cross-sectional transcriptome studies, it was suggested that most transcriptional alterations in brain frontal cortex occur around the age of 42 [10], and in Vastus lateralis muscle major changes occur already in the fourth decade [11]. In both studies, two linear regression models were applied to identify the age-positions. Applying a quadratic regression model we indeed confirmed that major expression profiles are changed first in the fourth decade in both brain frontal cortex and Vastus lateralis muscle [9].

Ideally, the pattern of aging-associated molecular changes could be extracted from population-based datasets [12]. These datasets are cross-sectional, covering a broad age-range, and all subjects are included. Most populationbased datasets are skewed in the old age, making a linear regression model unfit. Here we investigated ageassociated molecular changes in whole blood from two population datasets. The Rotterdam Study (RS) cohort III [13] and the SHIP-TREND cohort [14, 15] were independently generated using RNA microarrays. After correcting for the skewed sample distribution across age, we demonstrate that an age-associated pattern of molecular changes is highly similar between the two datasets. We show that in whole blood major molecular changes occur only at the seventh decade, predominantly affecting the translation and immune cellular machineries.

\section{RESULTS}

Dataset demographics of the RS and the SHIPTREND are presented in Table 1. Each datasets was analyzed independently and subsequently compared for replication, confirmation and identification of the most consistent age-dysregulated genes. In both studies, all subjects are Caucasian. Gender distribution across age was similar and not skewed in both datasets (Supplementary Table S1). Outliers were not identified by using principal component analysis (Supplementary Figure S1). Therefore, all subjects were included and the models were not corrected for potential confounders. However, the sample distribution over age and the age-range differed between the two studies (Table 1 and Supplementary Figure S2), and a positive skewness was found in the RS dataset, indicating underrepresentation of elderly subjects (Supplementary Figure S2). To compensate for the uneven sample distribution we included a sample-weighting step, which downsized the effect of overrepresentation and underrepresentation in the population. Sample weighting was achieved using moving decade age groups inversely proportional to the size of their neighbourhood. We compared the number of significantly dysregulated probes ( $p$-value $<0.05$; false discovery rate (FDR)) between the non-weighted and weighted datasets and found that the percentage of overlapping age-associated genes between the weighted and non-weighted datasets was high for both the RS and SHIP-TREND dataset (76.7\% for RS, $94.4 \%$ for SHIP-TREND; Table S2). Higher percentage of overlapping genes in the SHIP-TREND dataset is expected due to a more even distribution with age as compared to the RS dataset (Supplementary Figure S2). A higher number of genes were identified as age-associated after sample weighting compared with the non-weighted, in both datasets (Supplementary Table S2).

The age-associated probes were identified using a basis spline (b-spline) quadratic regression model. To reduce assumptions in the model, the b-spline excluded control knots [9]. Significant probes were considered with a $p$-value $<0.05$ (FDR). Out of all probes, $73 \%$ and $41.7 \%$ were significantly age-associated in the RS and in the SHIP-TREND dataset, respectively, however most significant probes $(93.3 \%$ and $95.4 \%$; (Supplementary Table S2)) had a fold change (FC) $<1.2$ in absolute value. As fold change is crucial to assess the rate of changes over age, we applied a FC filter. We selected a FC $\geq 1.2$ in absolute value as a threshold, based on a recent study suggesting that this numeric FC value renders more reproducible results [16].

The probes that passed the FDR $<5 \%$ and absolute $\mathrm{FC} \geq 1.2$ filtering criteria, 1023 in the RS and 990 in SHIPTREND (Table 2) were clustered using $K$-means clustering method. $K$-means with Euclidean distance as a distance measure was used to identify the age-associated trends in the expression profile datasets, with the age being used as a continuous variable, and thus rendering it most suitable for the analysis [9]. Clustering was performed on each dataset separately, and the most consistent clusters were identified with the significant overlapping genes between the two datasets. After merging the clusters with redundant trends and exclusion of the clusters with $N=1$, in both datasets two major age-associated expression profiles with opposite inclinations were identified (Figure 1A).

To assess the age point at which the major changes in the expression profiles occur, $K$-means clustering using absolute correlation as a distance measure was applied. The age when major changes occur (i.e. age-position) was determined from the conjunction point between the opposite inclinations (Figure 1B). Importantly, only one age-position was identified in either dataset. In the RS dataset, the age-position occurred at the end of the seventh decade and in the SHIP-TREND dataset in the beginning of the seventh decade (Figure 2B). The earlier occurrence of the age-position in SHIP-TREND compared with RS could be attributed to the difference in the age ranges of the datasets. In a previous study, we showed that the ageposition is influenced by the age range of the dataset [9]. Therefore, we then verified the age-position in matched age range (46 to 81 years of age) datasets. The whole analysis was repeated in the age-matched datasets, and 296 probes were filtered as significant in the RS, and 857 probes in the SHIP-TREND. Also in these datasets only 
Table 1: Study demographics

\begin{tabular}{lcccc}
\hline & Samples & Age range & "Probes & "Annotated probes \\
\hline RS & 762 & $46-89$ & 21238 & 15216 \\
SHIP-TREND & 991 & $21-81$ & 48803 & 24928 \\
Overlap (\%) & & & $19750(93 \%)$ & $13741(90 \%)$ \\
\hline
\end{tabular}

Table shows the number of included study participants, age range in years, number of probes and number of annotated probes (using Entrez gene ID) for the RS and the SHIP-TREND dataset. The overlap between RS and SHIP-TREND is calculated out of the RS dataset.

one age-position was found, and it was mapped to the seventh decade in both RS and SHIP-TREND datasets (Supplementary Table S3 and Figure S3). This indicates that the difference in the occurrence of the age-position between the full and the age-matched datasets is due to the different age range covered by the two studies. Moreover, in the RS dataset only $21 \%$ of the genes that passed the $p$-value and fold change filters were found in the age-matched dataset. This suggests that most gene expression changes are denoted by the age group $>81$ years old.
Subsequently, major age-associated molecular signatures in both datasets were identified using clustering of the gene functional groups into enrichment maps. Overall, enrichment maps in both datasets were highly similar, and the most prominent functional gene networks overlapped between the RS and the SHIPTREND. Reduced expression levels were found for genes of RNA metabolism and translation groups, and an increase in expression levels was found for genes of the defense response and erythrocyte system (Supplementary Figure S4). Gene networks of the immune system were

A

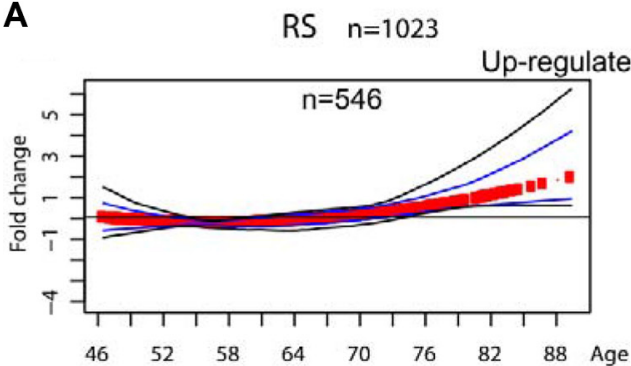

SHIP $n=990$

Down-regulated inclinations
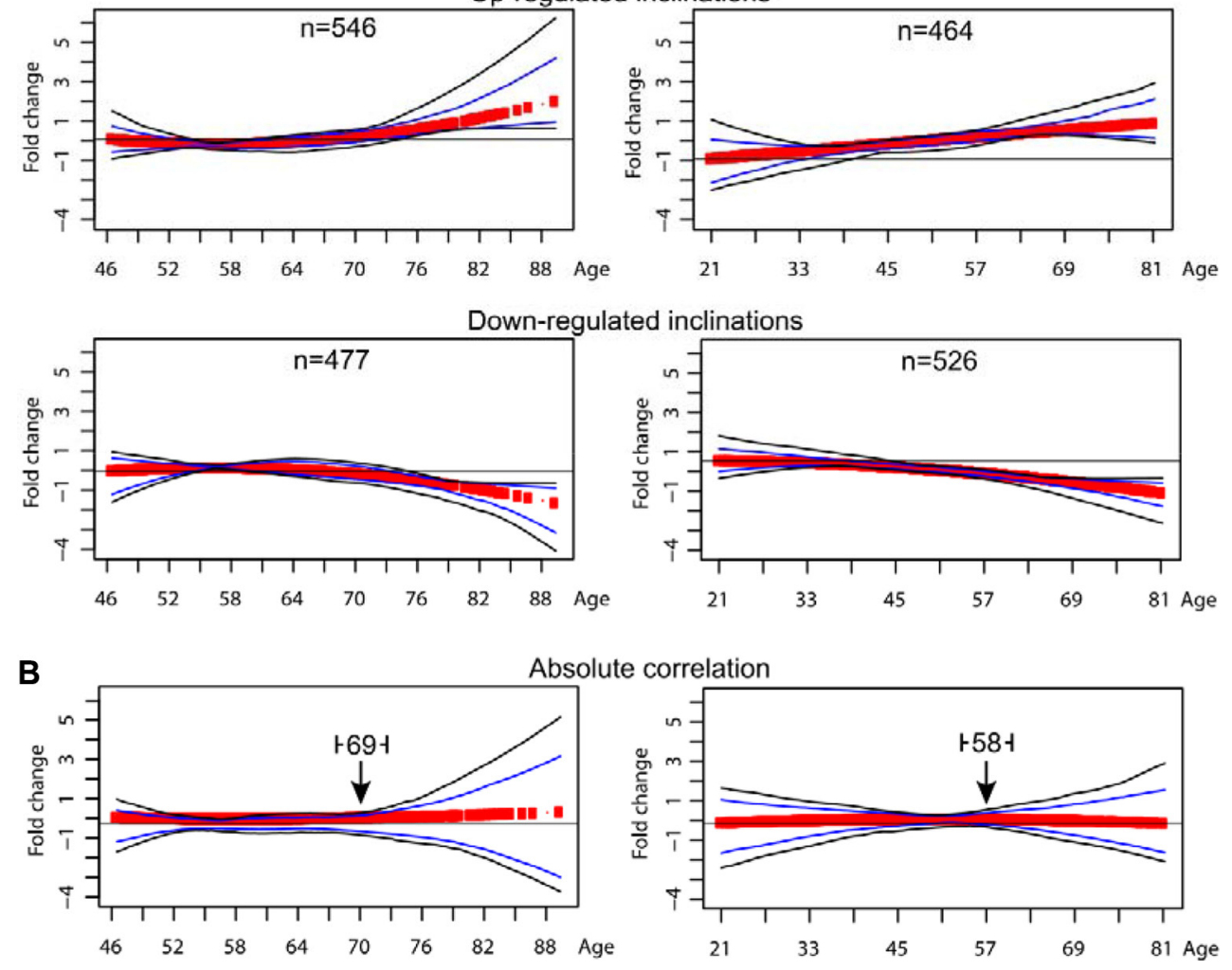

Figure 1: Trends of the clustered age-associated significant probes. Scatter plots showing the trends of the mRNA expression profiles of the age-associated significant probes clustered using $k$-means algorithm. Clustering includes only probes with FDR $<0.05$ and $\mathrm{FC} \geq 1.2$ in absolute value. Panel (A) shows the clusters identified using Euclidean distance as metric in the clustering algorithm, in the RS dataset (left) and in the SHIP-TREND dataset (right). The number of probes per cluster is depicted on top of every plot. Panel (B) shows the results of the $k$-means clustering using absolute correlation as distance metric. This facilitates the grouping of the probes presenting a symmetric expression profile. The arrowhead indicates the identified age-position in the RS dataset (left) and in the SHIP-TREND dataset (right). 
dysregulated without a specific dysregulation direction (Supplementary Figure S4).

The most consistent genes and gene networks were then identified by overlapping the significant probes between the two initial datasets. In total 378 dysregulated genes overlapped between the two datasets and for 375 genes dysregulation direction was the same as in the parental dataset (Figure 2A). The overlapping probes were enriched in the immune and defense responses, and in the translation machinery groups (Figure 2B). The genes associated with the translation network showed an age-associated down-regulation, whereas genes associated with the immune system presented a higher expression level with age (Figure 2B).

Next, we verified the molecular changes during the age-position. The RS dataset was split into two subsets with the age of 65 years being selected as a cut-off point. At 65 years the age-position was found in the two agematched datasets (Supplementary Table S2). The younger group $(<65$ years) comprised 606 individuals, whilst the older group ( $\geq 65$ years) included 156 individuals (Supplementary Table S4). In the younger group, only 128 probes were found to be significantly age-associated and those were not enriched in any functional group (Figure 2C). Those probes were not found among the overlapping genes between RS and SHIP-TREND datasets (Supplementary Table S5). This suggests that molecular changes in blood prior to 65 years are neither robust nor consistent. In contrast, in the older age group 1319 probes were age-associated (FDR $<5 \%$ and absolute $\mathrm{FC} \geq 1.2$ ) and those were mapped to the immune system, translation and the defense response functional groups (Figure 2C). $65 \%$ of those genes overlapped with the significant probes from the SHIP-TREND (Supplementary Table S5). This indicates that major expression profile alterations in blood occur from the seventh decade onwards. This procedure was conducted in the RS study only as less subjects of older age are found in the SHIP-TREND dataset.
A

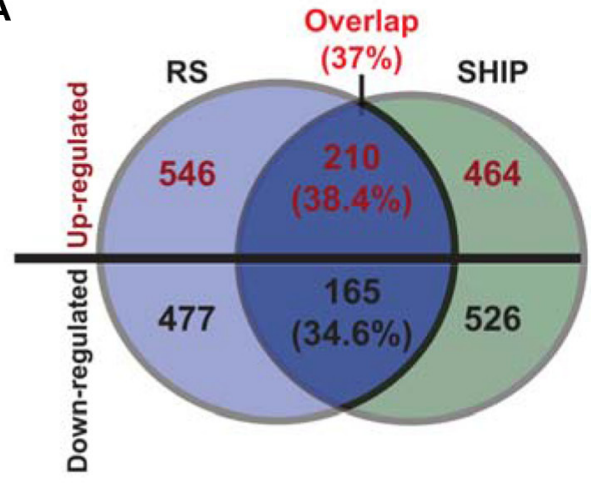

C

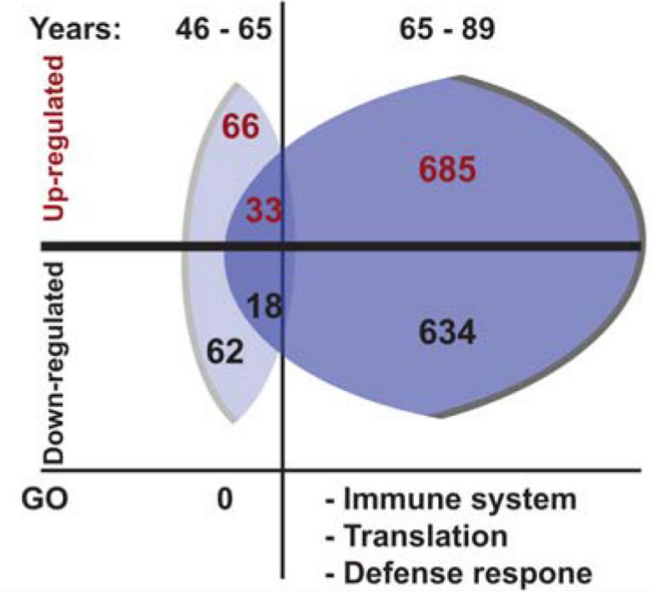

B

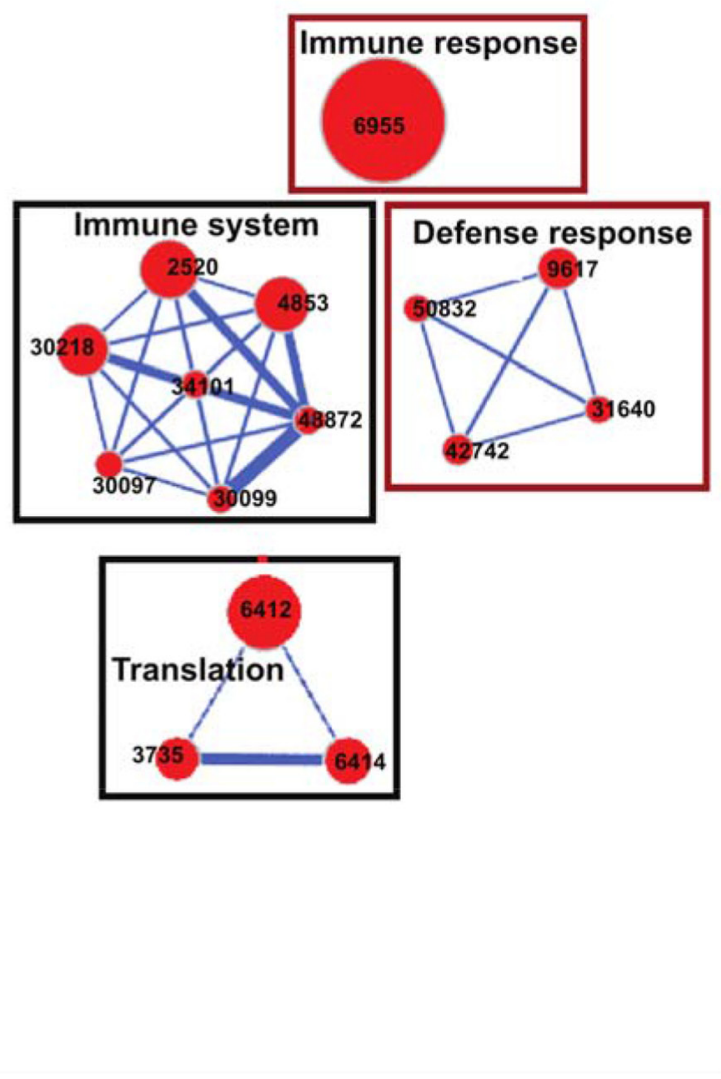

Figure 2: Gene network of the overlapping significant genes. (A) Venn diagram showing the overlap of the significant (FDR $<0.05$ and FC $\geq 1.2$ ) age-associated probes in RS (blue) and SHIP-TREND (green) datasets. Up- or down- regulated genes are depicted in red or black text colour, respectively. In parentheses, the probe overlap in percentages is indicated, out of the RS dataset. (B) Cytoscape enrichment maps of enriched Gene Ontology (GO) groups in the overlapping genes from RS and SHIP-TREND. GO groups are denoted with the ID number of each term, and the size of the nodes is proportional to the number of genes associated to the node. Gene networks are connected with blue lines, a thicker line representing a stronger connection. Down regulated gene modules are gated black and the up regulated are gated red. (C) A schematic representation of the dysregulated genes in datasets subdivided for 46-65 years and 65-89 years. Numbers in parentheses show the percentages from the overlapping genes (in A), and indicates up or down regulated genes in each age group. Gene network clusters for each age group are specified. 


\section{DISCUSSION}

Aging-associated molecular changes are highly prominent and are widespread across the genome. However, their progression and the rate of change are poorly understood, in part because linear models are most often applied for age-associated datasets. Here, we applied a quadratic model on two population-based datasets and found that in whole blood major expression profile changes occur during the seventh decade. This age-position was verified and was found to be robust and consistent. This age-position is in agreement with a recent study showing that the number of immune cells and T-cell receptor reduces from the seventh decade onwards [17]. Our analyses revealed that the most consistently and prominently affected gene networks are of the immune system and of the translation machinery. Those molecular signatures were also found in the RS dataset using a linear regression model [18]. We show that genes of the translation machinery showed reduced expression levels with age, suggesting reduced protein translation during aging in blood. Reduced total protein expression during the seventh decade was reported from proteomic studies in blood [19]. Moreover, slower rate of protein synthesis during aging was also reported $[20,21]$. This suggests that the age-associated expression profiles reflect agingassociated functional changes in blood.

Whether or not the rate of molecular aging is similar between tissues is poorly understood. In whole blood, we identified only a single age-position during the seventh decade. A single age-position was found in kidney cortex, also during the seventh decade [9]. However, in brain frontal cortex and in Vastus lateralis muscle two agepositions were identified, the first during the fifth decade and a second one during early eighth decade [9]. This suggests that in humans the age at which major molecular changes occur differs between tissues. This conclusion in agreement with physiological studies suggesting that the rate of age-associated tissue deterioration differs between tissues [8]. Moreover, the prominent aging-associated gene networks also differ between tissues: translation and the immune system gene networks from blood were not identified in brain cortex or skeletal muscles tissues [9]. An age-position could indicate an aging-associated disease risk for tissue-specific disorders and could be a consideration for treatments and interventions during aging.

This methodology holds several limitations. Despite the fact that it is able to overcome the differences between the dataset platforms and identify the age-positions at which major changes in expression profiles occur, we note that the results from a quadratic regression model can fluctuate due to variations in the age range covered by the dataset and the age-dependent distribution of the samples. This effect is acknowledged, explained and quantified in our previous study on two different datasets from brain frontal cortex and Vastus lateralis muscle [9].
In population-based studies, the subjects are often not selected or filtered. This can cause a bias in the results, if certain confounders are not evenly distributed across age. For example, subjects are likely to differ in health features, lifestyle and socioeconomic status. We did not identify major outliers, suggesting that socioeconomic differences may not have a major contribution to the transcriptome in whole blood. We did not find an uneven gender distribution across the age, in both datasets. This indicates that the age-associated pattern we report here would not be caused by differences in the gender distribution across age. However, molecular aging highly differs between genders [7]. How the age-position differs between genders should be addressed in future studies. We found that subject distribution is skewed in the old age, and applied sample weighting that compensates for the uneven sample distribution across age. With this procedure, two independent datasets can be compared [4]. Sample weighting resulted in an increased number of age-associated transcripts that pass the $p$-value threshold. However, the majority of transcripts had an absolute $\mathrm{FC}<1.2$. To increase selectivity, we applied a fold change filter, but a confident fold change threshold for aging studies should be determined in future studies using an independent procedure.

\section{MATERIAL AND METHODS}

\section{Datasets}

We performed a differential expression study in human peripheral blood samples from two crosssectional datasets from two independent large prospective, population-based cohort studies. The Rotterdam Study (RS) $[13,22]$ consists of an ethnically homogenous group of 762 Caucasian subjects aged 46-89 years in the district of Rotterdam, the Netherlands. The SHIP-TREND (Study of Health in Pomerania) [14, 15] contains 991 individuals with available gene expression and phenotype data aged 21 to 81 years from the German region of West Pomerania. The initial analysis was carried out in the RS dataset and replication was performed in the SHIPTREND dataset. RS participants (aged 46 years and older) were all examined in some detail at baseline: they were interviewed at home and then had an extensive set of examinations in a specially built research facility in the center of their district. These examinations were repeated every 3-4 years in characteristics that could change over time. The participants in RS are followed for a variety of diseases that are frequent in the elderly. The study has been approved by the Medical Ethics Committee of the Erasmus MC and by the Ministry of Health, Welfare and Sport of the Netherlands, implementing the Wet Bevolkingsonderzoek: ERGO (Population Studies Act: Rotterdam Study). All participants provided written informed consent to participate in the study and to obtain 
information from their treating physicians. Sample collection and data generation are detailed in [13]. The RS cohort III expression dataset is available at the GEO repository under the accession GSE33828.

The Study of Health in Pomerania (SHIPTREND) was conducted between 2008 and 2012. The SHIP population-based epidemiological study aims at investigating the risk factors of common, populationrelevant diseases. The study design and the sampling methods as well as genotyping and gene expression measurement and methods (Illumina HumanHT-12 v3 Expression Beadchips) have been described elsewhere $[14,15,23]$. The medical ethics committee of the University of Greifswald approved the study protocol, and oral and written informed consents were obtained from all study participants. The SHIP-TREND expression dataset is available at the GEO repository under the accession GSE36382: 991 samples are available for analysis.

Both gene expression datasets have been obtained using the Illumina HumanHT-12 Expression Beadchip, but on different microarray platforms: v3 for SHIPTREND and v4 for the RS-III respectively. The dataset demographics of both studies are summarized in (Table1).

\section{Pre-processing}

The gene expression levels were quantilenormalized and $\log 2$-transformed. Subsequently, probe and sample means were centered to zero. In the available RS dataset, probes were pre-filtered and declared significantly expressed when the detection $p$-values calculated by Illumina's GenomeStudio were $<0.05$ in more than $10 \%$ of all samples. For the SHIP-TREND dataset, there was no filtering of the samples prior to analyses, thus all probes were available for analysis (Table 1). No further correction for other covariates or diseases was performed on the datasets, as it is not the purpose of this study. In order to discard possible outliers, a principal component analysis (PCA) was performed on both datasets using the $R$-base packages of the statistical environment R (Supplementary Figure S1). Eventually, no samples were discarded. Both datasets equally passed all processing steps with the same parameter settings in the models involved, as described below.

\section{Sample weighting}

Prior to the selection of the most significant ageassociated probes, the samples were chronologically ordered. The RS dataset is skewed towards the elderly, thus the distribution of samples across age is uneven (Supplementary Figure S2). This is caused by the design of the RS cohort (RS-III is the youngest cohort of RS: most of the older individuals already participated in RS-I or RS-II). In order to compensate for this uneven distribution and reduce the likely influence upon the statistical tests, sample weighting [24] using a dynamic age window was applied using the following procedure: each sample was assigned to an age group that consisted of samples in the range of \pm 5 years from the age represented by the current sample. The weight value for each sample was calculated as being inversely proportional to the number of samples in its age group, following the equation:

$$
w_{i}=\frac{1}{N_{i}} x_{i}
$$

where $x i$ is a sample from the dataset and $N i$ represents the number of samples present in the age group of a sample:

$$
N_{i}=\sum_{(j=1)}^{n}\left[s_{j}=x_{i} \pm 5\right]
$$

with $s j$ being the number of samples $x i$ for subject $j$ in its designated age group.

\section{Probe smoothing}

In order to reduce the inter-individual variation and facilitate the identification of age associated expression trends, the data was smoothed probe-wise. To achieve this, a simple quadratic regression model was used. The motivation behind this choice and a detailed description of the model used can be found in [9]. The calculated weights for each sample represented a parameter in the regression model, following the equation:

$$
E_{i j}=\alpha_{i}=+\beta_{i}\left(x_{j} w_{j}\right)+\gamma\left(x_{j} w_{j}\right)^{2}+\varepsilon_{i j}
$$

where $E_{i j}$ is the intensity of probe intensity $\mathrm{i}$ for subject $j, x_{j}$ the age of subject $j, w_{j}$ the weight for subject $j, \alpha_{i}, \beta_{i}$, $\gamma_{i}$ are probe-specific regression parameters, and $\varepsilon_{i j}$ is the residual error.

\section{Probe filtering}

Filters were put in place in order to identify the significant age-associated probes. The $p$-value of each probe was calculated, testing the null hypothesis of no age association versus the quadratic regression model used in the prior step. The $p$-values were adjusted for multiple testing using the Benjamini-Hochberg false discovery rate (FDR) method. The probes presenting a FDR $<0.05$ were considered statistically significant and kept for further analysis. Due to the large number of probes passing the $p$-value threshold (Figure 3 and Supplementary Table S2), a second filter set a threshold on the absolute value of the fold change (FC) [25]. There is no consensus on a FC threshold, but literature shows that an absolute $\mathrm{FC} \geq 1.2$ renders the results more likely to be reproducible [16]. Therefore, only probes that presented a $\mathrm{FC} \geq 1.2$ in absolute value were kept for the subsequent steps of the analysis. 
Table 2: Trends of the significant probes

\begin{tabular}{lccc}
\hline \multicolumn{1}{c}{ Dataset } & Positive regulation & Negative regulation & Total \\
\hline RS & 546 & 477 & 1023 \\
SHIP-TREND & 464 & 526 & 990 \\
Overlap & 210 & 165 & 378 \\
\hline
\end{tabular}

Table shows the number of filtered (FDR $<0.05$ and absolute FC $\geq 1.2$ ) significant age-associated probes per trend of the expression profile, as well as the total for each of the datasets. The overlap of significant probes between the two datasets is also shown. The total overlap is higher than the sum of the overlapping probes, which present a positive regulation and of the probes presenting a negative regulation, as some probes can present a positive regulation in one of the datasets and a negative regulation in the other.
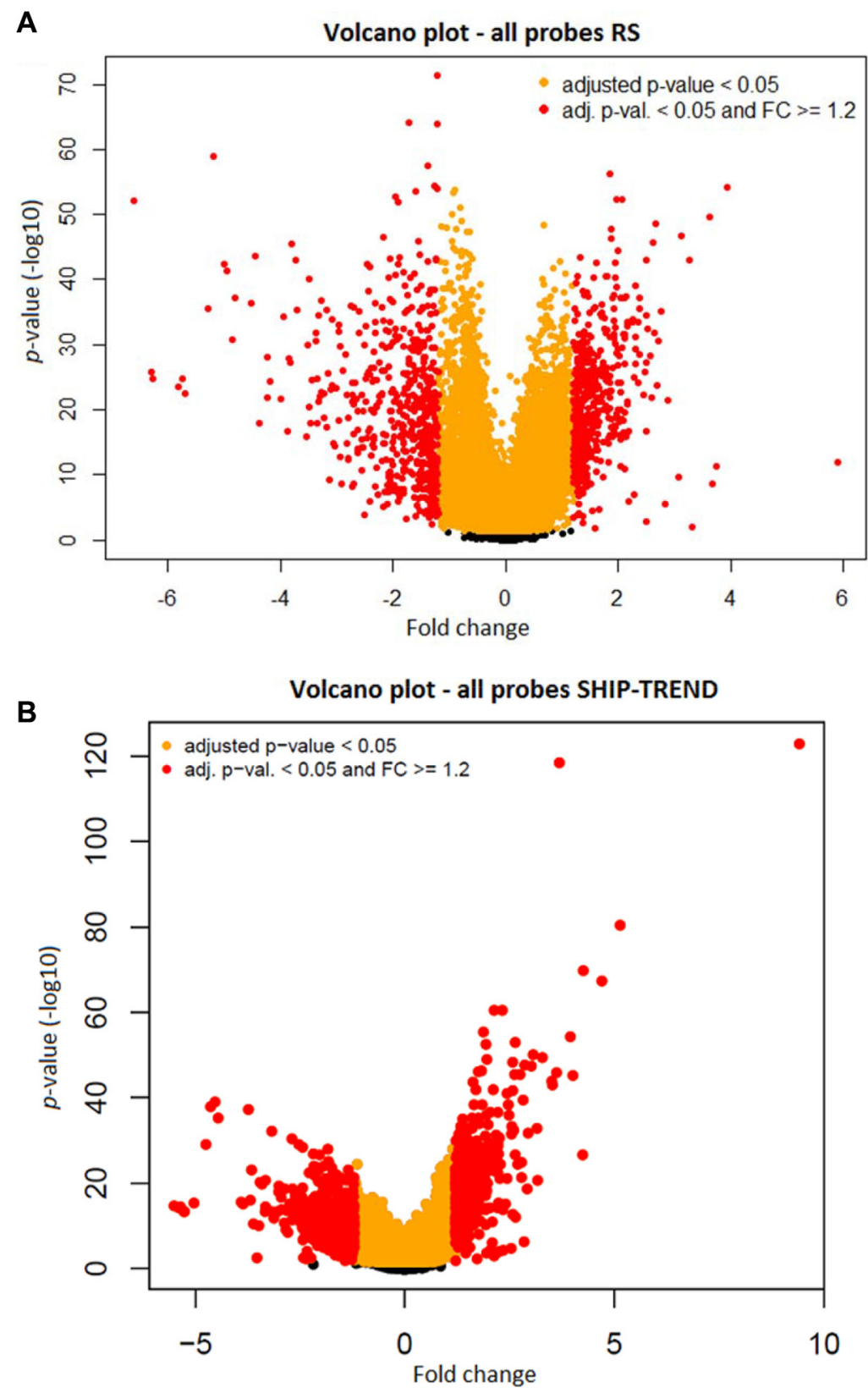

Figure 3: A volcano plot of all the probes from the RS dataset (A) and from the SHIP-TREND dataset (B):Volcano plots of the - $\log 10 p$-value (Y-axis) against the fold change (X-axis) show an association in the RS dataset (A) and in the SHIPTREND dataset (B). In yellow, the probes with FDR $<0.5 \%$ and in red, the probes with FDR $<5 \%$ and an absolute fold change $(\mathrm{FC}) \geq 1.2$. In black are depicted the probes that did not pass the prior filters. 


\section{Clustering}

The resulting set of significantly age-associated smoothed probes was standardized by subtracting the average intensity value of a probe from each of its data points. This allowed the probes to group based on the trend similarity rather than intensity. Grouping of the probes based on similarities in their expression profiles was achieved using $K$-means clustering as Euclidean distance measure of similarity. $K$-means clustering using absolute correlation as distance metric was used to identify the age-position. A detailed description of the clustering mechanism, as well as the statistical evaluation for confidence of the resulting clusters can be found in [9]. Major age-associated trends were identified after merging of the redundant clusters and exclusion of the single-gene clusters.

\section{Enrichment maps}

The enrichment analysis was performed using the Entrez gene identifiers. The mapping of the probes to their Entrez gene identifiers was made with Illumina Human HT-12 v3 and v4 arrays platforms. From the significant probes, $2.8 \%$ were without annotation and were excluded. Enrichment maps were carried out using the entire set of probes as background in the DAVID 6.7 online analysis tool [26]. DAVID 6.7 analyses of the molecular functions and the biological processes of the gene ontology (GO) were performed. The analysis was carried out for each dataset separately, on the entire set of significant probes, as well as for the up or down regulated probes separately. Subsequently, the significant ( $p<0.05$, FDR) GO terms were used as input in the Cytoscape 3.2.0 open source platform [27] and enrichment maps were created for upand down-regulated genes separately.

\section{Supporting Data}

The entire list of significantly age-associated genes for each dataset, as well as a list of the overlapping significant genes between the two datasets can be found in text format as supplementary material (Supplementary Tables S6-S8). These lists include the detected $p$-value after FDR, and the direction of regulation of each probe.

\section{Accession numbers}

The accession number for the RS cohort III dataset used in this paper is GEO:GSE33828.

The accession number for the SHIP-TREND dataset used in this paper is GEO:GSE36382.

\section{ACKNOWLEDGMENTS}

We Thank Jelle Goeman for a bio-statistical advise. This study is supported, in part, by AFM-TELETHON.
The Rotterdam Study is funded by Erasmus Medical Center and Erasmus University, Rotterdam, Netherlands, Organization for the Health Research and Development (ZonMw), The Netherlands Organisation of Scientific Research NWO Investments (nr. 175.010.2005.011, 911-03-012), The Research Institute for Diseases in the Elderly (014-93-015; RIDE2), The Ministry of Education, Culture and Science, The Ministry for Health, Welfare and Sports, the European Commission (DG XII), and The Municipality of Rotterdam. The generation and management of RNA-expression array data for the Rotterdam Study was executed and funded by the Human Genotyping Facility of the Genetic Laboratory of the Department of Internal Medicine, Erasmus MC, The Netherlands. We thank Ms. Mila Jhamai, Ms. Jeannette M. Vergeer-Drop, Ms. Bernadette van Ast-Copier, Mr. Marijn Verkerk and Jeroen van Rooij, BSc for their help in creating the RNA array expression database. MP and JvM were funded by The Netherlands Society for Scientific Research (NWO) VIDI Grant 917103521.

SHIP-TREND: SHIP is supported by the BMBF (German Ministry of Education and Research) and by the DZHK (Deutsches Zentrum für Herz-KreislaufForschung - German Centre for Cardiovascular Research). SHIP is part of the Community Medicine Research net of the University of Greifswald, Germany, which is funded by BMBF (grants no. 01ZZ9603, 01ZZ0103, and 01ZZ0403), the Ministry of Cultural Affairs as well as the Social Ministry of the Federal State of MecklenburgWest Pomerania, and the network 'Greifswald Approach to Individualized Medicine (GANI_MED)' funded by the BMBF (grant 03IS2061A). Generation of genomewide data has been supported by the BMBF (grant no. 03ZIK012).

The authors are very grateful to the study participants, the staff, the general practitioners, and the pharmacists from the Rotterdam Study and the SHIPTREND cohort.

\section{CONFLICTS OF INTEREST} Interests.

All authors declare no Competing Financial

\section{Authors' contributions}

Analyses were carried out by Marius Gheorghe and Claudia Schurmann, study design by Vered Raz and Marius Gheorghe. Datasets were generated and contributed to by Joyce B.J. van Meurs, André G. Uitterlinden, Marjolein J. Peters, Albert Hofman, Claudia Schurmann, Reiner Biffar, Georg Homuth and Uwe Volker. The manuscript was written by Marius Gheorghe and Vered Raz. All authors read and commented on the manuscript. 


\section{REFERENCES}

1. Vijg J, Suh Y. Genetics of longevity and aging. Annu Rev Med. 2005; 56:193-212.

2. Chen HY, Maklakov AA. Longer life span evolves under high rates of condition-dependent mortality. Curr Biol. 2012; 22:2140-2143.

3. Abrams PA. Does Increased Mortality Favor the Evolution of More Rapid Senescence? Evolution. 1993; 47:877-887.

4. Williams PD, Day T. Antagonistic pleiotropy, mortality source interactions, and the evolutionary theory of senescence. Evolution. 2003; 57:1478-1488.

5. Glass D, Vinuela A, Davies MN, Ramasamy A, Parts L, Knowles D, Brown AA, Hedman AK, Small KS, Buil A, Grundberg E, Nica AC, Di Meglio P, et al. Gene expression changes with age in skin, adipose tissue, blood and brain. Genome Biol. 2013; 14:R75.

6. Gonzalo S. Epigenetic alterations in aging. Journal of applied physiology (Bethesda, Md : 1985). 2010; 109:586-597.

7. Hannum G, Guinney J, Zhao L, Zhang L, Hughes G, Sadda S, Klotzle B, Bibikova M, Fan JB, Gao Y, Deconde R, Chen M, Rajapakse I, et al. Genome-wide methylation profiles reveal quantitative views of human aging rates. Molecular cell. 2013; 49:359-367.

8. Yashin AI, Arbeev KG, Wu D, Arbeeva LS, Kulminski A, Akushevich I, Culminskaya I, Stallard E, Ukraintseva SV. How lifespan associated genes modulate aging changes: lessons from analysis of longitudinal data. Frontiers in gen. $2013 ; 4: 3$.

9. Gheorghe M, Snoeck M, Emmerich M, Back T, Goeman JJ, Raz V. Major aging-associated RNA expressions change at two distinct age-positions. BMC Genomics. 2014; 15:132.

10. Lu T, Pan Y, Kao SY, Li C, Kohane I, Chan J, Yankner BA. Gene regulation and DNA damage in the ageing human brain. Nature. 2004; 429:883-891.

11. Anvar SYea. A decline in PABPN1 induces progressive muscle weakness in Oculopharyngeal muscle dystrophy and in muscle aging. Aging (Albany, NY). 2013; 5:412-426. doi: 10.18632/aging.100567.

12. Zahn JM, Poosala $\mathrm{S}$, Owen $\mathrm{AB}$, Ingram DK, Lustig A, Carter A, Weeraratna AT, Taub DD, Gorospe M, MazanMamczarz K, Lakatta EG, Boheler KR, Xu X, et al. AGEMAP: a gene expression database for aging in mice. PLoS Genet. 2007; 3:e201.

13. Hofman A, Brusselle GG, Darwish Murad S, van Duijn CM, Franco $\mathrm{OH}$, Goedegebure A, Ikram MA, Klaver CC, Nijsten TE, Peeters RP, Stricker BH, Tiemeier HW, Uitterlinden AG, Vernooij MW. The Rotterdam Study: 2016 objectives and design update. Eur J Epidemiol. 2015; 30:661-708.

14. John U, Greiner B, Hensel E, Ludemann J, Piek M, Sauer S, Adam C, Born G, Alte D, Greiser E, Haertel U, Hense HW, Haerting J, et al. Study of Health In Pomerania (SHIP): a health examination survey in an east German region: objectives and design. Sozial- und Praventivmedizin. 2001; 46:186-194.

15. Volzke H, Alte D, Schmidt CO, Radke D, Lorbeer R, Friedrich N, Aumann N, Lau K, Piontek M, Born G, Havemann C, Ittermann T, Schipf S, et al. Cohort profile: the study of health in Pomerania. Int J Epidemiol. 2011; 40:294-307.

16. St Laurent G, Shtokalo D, Tackett MR, Yang Z, Vyatkin Y, Milos PM, Seilheimer B, McCaffrey TA, Kapranov P. On the importance of small changes in RNA expression. Methods (San Diego, Calif). 2013; 63:18-24.

17. Vallejo AN. Immune remodeling: lessons from repertoire alterations during chronological aging and in immunemediated disease. Trends in Mol Med. 2007; 13:94-102.

18. Peters MJea. The transcriptional landscape of age in human peripheral blood. Nat Com. 2015; 6:8570.

19. Ignjatovic V, Lai C, Summerhayes R, Mathesius U, Tawfilis S, Perugini MA, Monagle P. Age-Related Differences in Plasma Proteins: How Plasma Proteins Change from Neonates to Adults. PloS one. 2011; 6:e17213.

20. Van Remmen H, Ward W, Sabia R, A R. Gene Expression and Protein Degradation. Comp Physiol. 2011; Suppl 28:171-234.

21. Tavernarakis N. Ageing and the regulation of protein synthesis: a balancing act? Trends in Cell Biol. 18:228-235.

22. Westra HJ, Peters MJ, Esko T, Yaghootkar H, Schurmann C, Kettunen J, Christiansen MW, Fairfax BP, Schramm K, Powell JE, Zhernakova A, Zhernakova DV, Veldink JH, et al. Systematic identification of trans eQTLs as putative drivers of known disease associations. Nat gen. 2013; 45:1238-1243.

23. Schurmann C, Heim K, Schillert A, Blankenberg S, Carstensen M, Dorr M, Endlich K, Felix SB, Gieger C, Grallert H, Herder C, Hoffmann W, Homuth G, et al. Analyzing illumina gene expression microarray data from different tissues: methodological aspects of data analysis in the metaxpress consortium. PloS one. 2012; 7:e50938.

24. Dumouchel WHD, Greg J., Ismael. Using Sample Survey Weights in Multiple Regression Analyses of Stratified Samples. Journal of the American Statistical Association. 1983; 78:535-543.

25. Xinmin L, Kim J, Zhou J, Gu W, Quigg R. Use of signal thresholds to determine significant changes in microarray data analyses. Genetics and Mol Biol. 2005; 28:191-200.

26. Dennis G, Jr., Sherman BT, Hosack DA, Yang J, Gao W, Lane HC, Lempicki RA. DAVID: Database for Annotation, Visualization, and Integrated Discovery. Genome Biol. 2003; 4:3.

27. Shannon P, Markiel A, Ozier O, Baliga NS, Wang JT, Ramage D, Amin N, Schwikowski B, Ideker T. Cytoscape: a software environment for integrated models of biomolecular interaction networks. Genome research. 2003; 13:2498-2504. 